\title{
Mariage humanitas-techne vers une culture de l'interculture
}

Jean-Marc Fick

\section{(2) OpenEdition}

1 Journals

Édition électronique

URL : http://journals.openedition.org/communicationorganisation/2762

DOI : 10.4000/communicationorganisation. 2762

ISSN : $1775-3546$

Éditeur

Presses universitaires de Bordeaux

Édition imprimée

Date de publication : 1 novembre 2002

ISSN : 1168-5549

Référence électronique

Jean-Marc Fick, " Mariage humanitas-techne vers une culture de l'interculture », Communication et organisation [En ligne], 22 | 2002, mis en ligne le 27 mars 2012, consulté le 19 avril 2019. URL : http:// journals.openedition.org/communicationorganisation/2762; DOI : 10.4000/

communicationorganisation. 2762

Ce document a été généré automatiquement le 19 avril 2019

(c) Presses universitaires de Bordeaux 


\title{
Mariage humanitas-techne vers une culture de l'interculture
}

\author{
Jean-Marc Fick
}

Il est question ici de soutenir tout de go le bienfait de l'interculturalité, croisement dû parfois au hasard, le plus souvent à une volonté ou à une nécessité de rencontre. On aborde en effet assez rarement la complémentarité des cultures, alors qu'on s'attache - le thème de la présente revue n'est pas exceptionnel - à déceler "la part de l'illusion ", résultant de risques possibles d'aliénation et d'appauvrissements identitaires.

2 Notre propos, résolument optimiste, puisqu'il se termine sur la perspective de l'avènement d'une véritable culture de l'interculture, ne fait pas référence au domaine de l'entreprise ou de l'institution, mais s'intéresse à une forme organisationnelle tout de même, celle de l'activité intellectuelle contemporaine. S'agissant de la rencontre entre la culture humaniste actuelle - qui englobe le domaine littéraire et artistique - et de la culture technologique - qui s'affirme avec l'expansion irréversible du numérique - nous verrons qu'il n'est pas illusoire, en effet, de prétendre que ces cultures se marient sous le régime de l'intérêt mutuel et donnent naissance à de nouvelles formes, riches et originales. L'hyperlittérature, comme la technimage, ou l'infothéâtralité, par exemple, sont le résultat d'un dialogue fécond entre l'homme et la machine. Par bonheur, la logique rationalisatrice de l'informatique binaire n'est pas venue rigidifier les processus et les langages, elle sait coexister avec la raison multiple qui anime l'homme.

3 Au préalable, posons bien que la technologie est une culture, à partir du moment où il est admis qu'une culture est une manière par laquelle s'exprime la nature humaine. Avec Malinowski, nous reconnaissons que tout ce qui est humain, les croyances, les rites, les normes, les idées, les valeurs, les arts et les coutumes, est partie intégrante de la culture, mais il faut ajouter les langages de l'informatique, qui, parce qu'ils démultiplient le pouvoir de l'homme, suscitent chez les chercheurs des interrogations sur les performances créatives et symboliques, les registres de pensée, les comportements, les imaginaires, les nouvelles formes de la subjectivité et de l'identité. 

donc matière à s'enrichir au contact des " humanités », socle de la mesure, du classicisme, de la logique linéaire et culte pour ce qui est de l'homme exclusivement. Parallèlement, les humanistes s'ouvrent à des réflexions neuves et à des pratiques nouvelles en rencontrant les outils logiciels et les problématiques de relation, de transmission, d'expression que ces outils et leur usage génèrent.

Dans les universités, dans les lycées, comme dans les nombreuses entreprises tournées vers la création, ce n'est pas une pluriculturalité qu'on observe, mais une véritable interculture de l'intelligence et du savoir qui est en train de se construire, pour le bonheur de ceux des étudiants littéraires qui ont un flair suffisant pour pressentir que leur avenir professionnel s'inscrira dans une voie qu'on pourrait appeler celle des Nouvelles Humanités.

6 Ces allégations participent de l'idée générale largement admise selon laquelle les mécanismes socio-cognitifs de rejet, souvent renforcés par un ethnocentrisme latent, ne s'accélèrent pas forcément au contact de l'altérité, que les échanges et l'hybridation revivifient en fait les cultures. D'ailleurs, toutes les cultures ne sont-elles pas le résultat d'une permanente fécondation mutuelle? L'interculturel a toujours existé car la rencontre des cultures a toujours eu lieu et a sûrement permis à l'humanité d'évoluer. On sait bien qu'aucune culture n'est restée à l'identique au cours des siècles. Les différences sont en vérité moins des facteurs d'opposition que d'enrichissement, à condition que s'harmonisent le principe d'identité et celui de métissage. Toutefois l'interculturalité ne va pas toujours de soi, il convient donc, préalablement, de faire le point sur les affects qui accompagnent particulièrement la culture technologique, la plus récente, donc la plus sujette à la méfiance. Ce sont des mouvements d'attirance ou de rejet, où le rationnel a sa part, mais pas exclusivement.

\section{À l'égard de l'autre culture : rejet ou sympathie}

7 Toute entreprise est interculturelle à partir du moment où elle cherche à construire, pour reprendre la formule de Carmel Camilleri, « une articulation entre porteurs de cultures différentes, visant à prévenir les inconvénients de leur coexistence et à les faire bénéficier des avantages qui en sont attendus ». Les modalités pratiques de réussite sont multiples et dépendent des acteurs engagés. "Ce ne sont jamais », en effet, « les cultures qui se rencontrent, mais leurs porteurs, qui, par leur dynamisme, y introduisent de nouveaux paramètres ${ }^{1}$ ».

8 Malgré le dynamisme des "porteurs", une rencontre ou un projet interculturel ne se réalise pas sans difficultés, parce que les frontières symboliques sont parfois épaisses.

9 La technologie, en l'occurrence, a toujours suscité méfiance et rejet: depuis la mise en garde platonicienne de Teuth jusqu'à un certain courant technophobe actuel qui annonce, devant le développement d'une réalité virtuelle, une nouvelle «blessure narcissique ${ }^{2}$ » de l'humanité (après celles causées par les révélations de Copernic, Darwin et Freud) ou qui augure d'une intelligence artificielle si développée que les ordinateurs nous accepteront près d'eux uniquement comme animaux de compagnie.

10 La culture technologique a effrayé et effraie encore l'employé de bureau, la secrétaire ou le professeur parce que son terrain est complexe, que «les paramètres techniques sont bien loin d'être les seuls...: ils se combinent avec du symbolique, des représentations 
mentales, des pratiques sociales...». ainsi que le remarque justement Jean-Baptiste Carpentier 3 . C'est ainsi qu'on répète à l'envi que. « dès que le premier tracteur est apparu, le chant du laboureur a disparu $»^{4}$. Il est vrai qu'on peut aussi être sensible à toutes les pathologies de la médiation technique qui portent atteinte au réel, à ces phénomènes qui font que l'individu se "cybiontise ${ }^{5}$, que l'existence prend une double forme, réelle et virtuelle. Pour les philosophies spiritualistes, l'idée que la machine dotée par l'homme d'une forme d'intelligence puisse enrichir en retour l'homme est une idée qui heurte; notre pensée est autosuffisante, elle se dispense de levier technique. En Occident domine cependant un sentiment médiophile qui relève d'une tradition de pensée bien antérieure au modèle mécaniste cartésien ${ }^{6}$. La philosophie finira-t-elle par clore le débat ? La tentation de se dépouiller de toute prothèse technologique et de réinventer L'HOMME NU surgit inéluctablement à certaines époques et touche de nombreux contemporains. Au contraire, peu enclin à la technophobie, l'humaniste qui croit aux bienfaits de l'interaction hommes machines, peut voir dans l'interconnexion mondiale la représentation de valeurs du siècle des Lumières fondées sur les échanges de connaissances, sur l'égalité des relations humaines ${ }^{7}$. Devant le problème de la conscience universelle et de l'intelligence collective, il évoque le cosmopolites, modèle des cyniques stoïciens. L'humaniste internaute, honnête homme, poli et qui sait vivre en harmonie avec lui-même, les autres et la machine, supporte la modification des repères classiques parce que les principes qui régissent l'artefact et influent sur les modalités de l'échange sont de mieux en mieux conçus pour être plus proches de l'homme, de sa pensée, de sa logique, de sa sensibilité. Les interfaces intelligentes commencent en effet à " apprendre » les usagers, et non plus l'inverse ${ }^{8}$. Prendrait-on durablement conscience que la finalité de la vie n'est pas la tekhnê ? Naguère, Seymour Papert, dans Le jaillissement de l'esprit ${ }^{9}$, enjoignait de former à la logique informatique, celle qui préside à la structure «psychique » de la machine intelligente. Les volontés politiques actuelles en faveur de cette orientation, fortement généralisées dans le monde, semblent lui donner raison.

On peut somme toute maintenant affirmer qu'un rapprochement a eu lieu entre la culture et de la technique ${ }^{10}$. Si un tel écart a existé, rendant la rencontre difficile, c'est parce que la culture humaniste a toujours nié la réalité humaine dans la science technologique, comme le montre le philosophe Simondon ${ }^{11}$ C'était négliger le fait sans doute trop évident que les cultures et les systèmes de pensée perdurent pourtant grâce à des supports de transmission et à un langage de communication adéquat. L'humanisation actuelle des artefacts contribue au rapprochement.

12 On le constate sur le terrain des applications, le rapprochement des deux cultures porte ses fruits. L'alliance de la littérature et de l'ordinateur en est un exemple parmi d'autres, qui touche un domaine d'activités et de recherche en matière de fiction interactive, d'édition électronique ou d'hypertextualité littéraire.

\section{L'hyperlittérature}

13 En permettant un mode ${ }^{12}$ de lecture aléatoire et interactif, la fiction numérique hypertextuelle ou hyperlittérature rompt avec la tradition du récit linéaire qui dispose, ainsi que l'a défini Aristote ${ }^{13}$, d'un début, d'un milieu et d'une fin. "On passe». dit Guillaume Baudin. «du linéaire au combinatoire, d'un roman à lire à une œuvre à construire $»^{14}$. «On peut en un rien de temps, remarque aussi Michel Butor ${ }^{15}$ changer ce 
qu'on veut dans un texte, créer de multiples variantes. À partir d'une matrice, il est possible d'inventer des centaines d'histoires, des textes mobiles. »

L'hyperlittérature est ainsi née de la rencontre d'une tradition littéraire avec un nouveau support écranique de lecture. Ce qui a facilité la rencontre, et c'est là, d'une façon générale, une des conditions sine qua non de réussite d'un mariage interculturel, c'est la prédisposition d'une ou des deux cultures en contact. En l'occurrence, le principe de l'écriture hypertextuelle est en germe bien avant l'arrivée du numérique : il répond peutêtre même au mouvement naturel de la pensée humaine ${ }^{16}$. « Pourquoi premier chapitre ? Il serait aussi bien partout ailleurs. D'ailleurs, j'ai écrit le huitième chapitre avant le cinquième, qui est devenu ici le troisième ", avouait Charles Nodier dans Moi-même. Les recherches dadaïstes ou celles de groupes d'écrivains comme l'OuLiPo ont également préfiguré un grand nombre des composantes de l'art technologique actuel. Barthes parlait déjà de ses propres textes comme des «figures [qui] ne peuvent se ranger, s'ordonner, cheminer, concourir à une fin" et revendiquait, comme Foucault, "un lecteur qui ne soit plus seulement consommateur mais producteur de texte $»^{17}$.

Malgré l'abandon de la linéarité, de l'incipit et de la clôture unique du texte, la lecture trouve sens parce que, derrière l'écran, se trouve toute une technologie mise en place par un auteur bi culturellement compétent. De même que la poésie libère les mots de la contrainte linéaire pour les projeter dans une forêt de correspondances, l'hypertexte soustrait les séquences narratives aux règles du récit traditionnel pour les conduire dans un espace multidimensionnel. L'ordinateur invente des situations, manie la syntaxe, construit le temps, le lieu et la cohérence des personnages, organise des concepts. Le résultat de l'échange est loin d'être stérile comme on peut en juger par l'œuvre étincelante d'Anne-Cécile Brandenbourger, Apparitions Inquiétantes ${ }^{18}$. Il est même souvent riche, dans le sens où il est un spectacle, une mise en scène, une représentation s'adressant à tous les sens.

Les travaux des groupes littéraires (Laire, Alamo...), les ouvrages collectifs (Yann Queffelec), offrent des exemples de participation de la machine à la création de l'œuvre. D'autre part, l'animation de textes par des calligrammes ou des effets typographiques s'inscrit dans un courant poétique qui prend en compte la dimension matérielle du support d'écriture. Réunis par la revue électronique Alire, des poètes jouent sur une écriture en deux ou trois dimensions, sur les métamorphoses des lettres et des mots. La page-écran permet de donner au récit un rythme qui favorise des jeux de langage et des exercices de style associés au travail graphique du texte et de l'image.

Ces nouvelles formes artistiques sont nées d'une rencontre qui associe Tekhnê et Humanitas. La littérature apporte la créativité, des savoirs, des contenus, une longue expérience. La culture technologique, loin de se réduire au pouvoir d'un réseau et à des manipulations techniques, offre un "environnement de processus et de relations qui transforment les conditions intellectuelles de l'échange $»^{19}$. Elle provoque de nouvelles pratiques de lecture et d'écriture, de nouveaux modes d'expression, et, essentiellement, de nouveaux repères spatiaux et temporels.

En effet, l'écran, nouvel espace du texte, est un espace imaginaire qui « nourrit, écrit Olga Kisseleva, une nouvelle perception esthétique du monde réel». Autre particularité d'ordre spatial, la délocalisation, permettant à un texte d'être créé dans un lieu, lu en même temps dans un autre, et d'être transformé à distance. L'espace de l'œuvre hypertextuelle entretient ainsi d'étroites relations avec sa temporalité, tout à la fois 
valorisatrice de l'éphémère et quête d'infini. Avec les générateurs de texte conçus pour l'éphémère, «La signification passe par l'établissement de liens non préalablement fixés, par des réseaux d'indices. Le texte est «à faire », porteur de potentialités latentes que la participation du lecteur accepte ou non de révéler $»^{20}$. Le texte ne préexiste pas au programme qui l'engendre et il ne se constitue que dans le temps de sa lecture. S'il n'est pas imprimé ou enregistré, il retourne au néant. Parallèlement, il arrive que l'œuvre soit conçue pour ne jamais se figer. Chaque mot s'ouvre sur une infinité d'ailleurs. Un récit qui se déroule dans un présent éternel et c'est le monde qui réaffirme en permanence sa présence. Puisque "L'écriture appelle l'infini " " l'hypertexte en est le moyen d'accès, alors que le livre oblige à l'arrêt du texte.

Toutes les spécificités de l'hyperlittérature remettent ainsi en cause la définition du texte caractérisée principalement par son autonomie et par sa clôture. Le texte informatique se caractérise par une nouvelle textualité qui le rend mobile, instantané, interactif et délocalisé22.

En conséquence, l'écriture ne serait-elle pleinement écriture "que lorsqu'elle est pleinement technologie » (Jean-Pierre Balpe) ? Et la littérature se verrait-elle offrir par là même une chance de se renouveler ${ }^{23}$ ? En tout état de cause, si la constatation de Jacques Derrida. à savoir que «c'est dans la forme du livre que se laissent tant bien que mal engainer de nouvelles écritures, qu'elles soient littéraires ou théoriques ${ }^{24}$ est encore valable, il n'en reste pas moins que la rencontre des deux cultures a donné de nouvelles productions durables, marquant une révolution des humanités. Car il ne s'agit pas, comme on peut le penser de prime abord, d'une mode accordant la primauté de la démarche sur le résultat, du zapping sur la continuité, de la technique sur le contenu. Notre temps, en réconciliant l'homme et la machine, s'est durablement installé dans la culture de réseaux, de processus, de participation et d'interaction, pour une communication planétaire. L'hyperlittérature participe ainsi d'une idéologie prônant l'universel sans totalitée 25

\section{La technimage}

21 L'alliance Humanitas et Tekhnê est productive dans bien d'autres domaines, que nous ne pouvons tous parcourir dans un article. L'art visuel numérique, par exemple, moyen d'expression du technoartiste omniprésent, omnichronique, retrouve et enrichit la veine inventive des Ready Made surréalistes et des courants cinétiques. Un nouveau régime du voir s'installe où vacillent les codes de la disposition balisant les itinéraires du regard, où s'évanouit la problématique antique de la mimesis. Deleuze ${ }^{26}$ avait vu juste : «Cerveauinformation remplace Oeil-Nature ». Les notions d'objet, de concept et d'expérience se trouvent redistribuées, explique Alain Renaud-Alain, «par un mode d'approche capable de conjuguer, comme deux scènes complémentaires homologues, les deux mondes jusque-là concurrents : l'intelligible et le sensible $»^{27}$.

22 Avec la technimage, la pipe de Magritte, symbole de la trahison de l'image, pourrait devenir une pipe qui se fume. L'icône prend maintenant sens par le moyen d'algorithmes, de pixels, de modems et de caméras. Sans matérialité, elle se crée de nouveaux référents et éclaire des aspects inconnus du réel. Ainsi, les «metaxu», hybridations instables, instantanées, fantasmagoriques ${ }^{28}$ montrent, selon Philippe Quéau, ce qui s'agite sans cesse dans la nature et dans les entrelacs des algorithmes logico-mathématiques. Miguel Chevalier crée des mouvements et des formes en exploitant les parasites techniques, 
comme la neige de l'écran télévisuel, ou compose une symphonie visuelle en n'utilisant que les chiffres 0 et I, comme pour matérialiser, avec humour, les flux d'information. Les créateurs réalisent, grâce aux gestes des spectateurs, des « environnements » d'images, lieux d'interaction entre l'ambiance physique et psychologique. Ils osent s'affranchir des référents du réel, mettent en scène le monde virtuel, en brisant les frontières entre les genres. Ils prouvent souvent que la collaboration artistique est possible pour exploiter les découvertes de la science et les exploits de la technologie. Lovers, par exemple, du groupe Dumb Type, est un spectacle multimédia sophistiqué et hybride, entre les arts du spectacle et les arts plastiques. Jeffrey Shaw offre au spectateur, assis sur un disque pivotant, une visite interactive de son "Virtual Muséum » ou une promenade à vélo virtuelle dans une ville imaginaire ${ }^{29}$. Les Physiochromies évolutives de Carlos Cruz-Diez, l' Univers électronique de Gregorio Vardanega, qui invite le public à intervenir sur une atmosphère intérieure colorée sont parmi les réalisations actuelles dont les effets optiques varient selon l'humeur et les gestes du visiteur. Elles réitèrent en cela des expériences artistiques de type cinétique, et se raccrochent, aidés par la procédure numérique, à l'art et à la philosophie de la métamorphose. Elles relèvent d'une attitude mentale, moins préoccupée d'imitation que de simulation, de monosémie que de polysémie et définissent une forme d'art "intermédiaire » qui traduit le bizarre et l'instable, qualités qui les rapprochent d'une esthétique néo-baroque.

On peut ainsi risquer un éloge de l'image numérique, réussite interculturelle, pour ce qu'elle apporte de positif à l'homme et à ses représentations malades d'un manque « d'images adéquates $»^{30}$.

\section{L'infothéâtralité}

Un rapprochement interculturel fécond s'est également opéré dans la conception technologique. Les principes qui président à la constitution actuelle de l'artefact homme machine se rapprochent du mode de logique humaine et sont même empreints d'une certaine théâtralité ${ }^{31}$, avec ce que le terme sous-entend d'humanisme et d'humanité.

Des mots du lexique reflètent l'humanisation de l'artefact et éloignent du sabir cyber. Certains ramènent à la belle langue française, mais aussi au langage du quotidien, théâtre de la vie. La Commission Générale de Terminologie et de Néologie a adopté32 " frimousse ", équivalent canadien de « binette ", pour signifier l'association facétieuse de quelques caractères typographiques évoquant un visage expressif. "Causette", également sur la liste officielle, poétise l'échange de messages qui s'affichent sur un écran. La rhétorique du courrier électronique développe l'hybridation linguistique, mais témoigne aussi du souci d'intégrer de l'humanité dans l'échange médiate, d'allure et de terminologie instrumentales. Dès lors, on ne peut considérer comme simple coïncidence qu'un logiciel comme Director ait choisi de fonctionner à l'aide d'une terminologie reflétant les propriétés saillantes d'entités cette fois spécialement dramaturgiques: " scène ", « script ", « distribution », « comportement ", « acteur ", « numéro d'acteur ».

Le mot « ordinateur", lui-même, choisi par le Professeur Legrand pour la traduction de « computer » renvoie surtout à l'idée d'une mise en ordre qui équivaut à une mise en scène. Les indices verbaux incitent à saisir ce qu'ils suggèrent, à savoir des formes de théâtralité inhérentes à l'artefact. 
d'activité mentale qui est sollicité dans le décodage multisémiologique de la représentation théâtrale. Un simple clic de la souris sur un élément hypertextuel ouvrant un nouvel écran est du même effet magique que l'ouverture du rideau de scène, que l'éclairage qui fait surgir du nouveau, que la métamorphose de l'objet, signifiant polyvalent et protéiforme, qui fait jaillir d'autres signifiés. Tout comme l'espace théâtral qui délivre "une pluralité de substances signifiantes ${ }^{36}$ qui se combinent et s'entrecroisent, la page-écran est une "polyphonie informationnelle ${ }^{37}$ » où surgissent, sous le clic ludique, des configurations de textes, d'images et de sons qui se rapprochent, s'éloignent, se juxtaposent ou se superposent. Alors, de même que l'ordre théâtral ne s'institue qu'après un long désordre, selon la méthode de Jouvet ${ }^{38}$, lorsque tous les possibles de jeu et de personnage ont été explorés, le désordre apparent s'organise, par étapes, vers l'ordre. La mémoire de l'arborescence hypertextuelle se construit au fil de la navigation qui devient, pour reprendre l'expression que Julia Kristéva applique au théâtre, une "pratique signifiante ». Le bon choix, qui mène au but recherché, découle souvent d'un tâtonnement. Cette représentation de l'action est vraiment à l'instar du travail théâtral. Ainsi le dispositif de l'artefact ne peut se concevoir d'une manière réductrice, comme un assemblage d'outils uniquement fonctionnels: une forme de théâtralité lui a été nécessaire, une harmonie magico-ludique, en quelque sorte, conférant, sans « bruit », de l'énergie et du sens à l'épaisseur de signes ».

La scénique du multimédia numérique va de la transposition du document abstrait en une réalité interactive. Elle résulte de choix esthétiques et de contraintes techniques, et s'élabore pour une part au cours de la session interactive, en fonction de la navigation de l'utilisateur. Cela est à mettre en parallèle avec l'acte théâtral. L'alchimie qui mène du texte à la scène publique est subordonnée aux prises de décision du metteur en scène. «Sous ses impulsions, chacun des éléments doit retentir comme un ton de l'orchestre de façon immédiate, compréhensible, dans des limites temporelles précises ", recommande Svoboda $^{39}$ en des termes qui rappellent les paradigmes de la communication définis par Bateson. La médiation virtuelle suppose une compétence identique, car le dispositif hypertextuel, objet semi-construit, pousse le lecteur à se situer habilement dans 
l'ensemble de liens et de nœuds. Elle suppose aussi une symétrie relationnelle. Une fois encore, l'interculturalité a joué un rôle et son influence n'est sans doute pas hasardeuse. En effet, un projet comme celui d'Artaud ${ }^{40}$ a fait son chemin dans les mentalités modernes : il est fondé sur un refus d'une dissymétrie de la relation humaine. Fusion de la vie et du théâtre, absorption de la salle dans la scène, d'une part. Fusion, confusion du réel et de sa représentation d'autre part.

La scénique promeut principalement l'expression du Jeu. L'artefact en action est un jeu, de même nature que le jeu de l'action théâtrale et les deux cultures se rencontrent pour exploiter toutes les vertus du jeu qu'on ne peut développer ici. L'internaute, comme le praticien de théâtre, se dote d'une identité de joueur pour la raison qu'en se branchant librement dans un espace-temps de communication virtuelle, il se met entre parenthèses, dans une situation différente de la vie sociale normale. Il devient autre parce qu'il adhère à la confrérie des connectés et qu'il réalise, selon son plaisir, des activités ludiques ou qui procèdent du ludique. Le "faire comme si» du jeu compose aussi une organisation sémiotique du plaisiri ${ }^{41}$. La théâtralité y prend place dans la mesure où il s'agit d'être pour le temps du jeu ailleurs ou autre chose que ce que je suis. Ce mimicry ${ }^{42}$ coïncide avec le simulacre théatral que réalisent le masque, le déguisement et le rôle. Dans le cadre virtuel, comme sur la scène, se déroule un jeu, jeu d'imitation, double jeu, jeu dans le jeu, jeu avec l'imprévu, s'effectuent des essais sans risques, se visitent tous les possibles, se vivent des situations où l'on accomplit symboliquement des actes de vie que l'on peut à loisir arrêter et reprendre.

Dans les deux cultures qui s'interprètent, scène et site, comédien et surfeur sont des termes quasiment interchangeables. La théâtralité ludique qui s'y exerce ouvre à une dynamique de communication, une pratique attrayante de l'altérité, une attitude distanciée, et à un plaisir collectif et individuel; elle développe parallèlement la spontanéité, la concentration et le pouvoir de créer.

Puisqu'un apport interculturel fonctionne dans les deux sens, il est possible, en retour, d'évaluer quelques apports de la scénation sur notre fonctionnement mental et sur le théâtre lui-même.

Le nouveau régime d'imaginaire et d'intelligibilité qui constitue l'artefact et gouverne l'outil, conçu de mieux en mieux comme complément et prolongement de la pensée humaine, rend paradoxalement la pensée humaine plus présente, plus dynamique et s'affirme comme puissant facteur de formation et de création. Non pas sclérosée, mais matricée, mise en forme autant que mise en scène, la pensée fonctionne par réflexivité : la part qu'elle mobilise pour dialoguer est objectivée, et lui revient sans cesse sanctionnée. La réflexivité est facilitée par une procédure d'utilisation informatique balisée, qui développe une logique de l'efficacité. Si, dans nos activités quotidiennes, nous tâtonnons dans l'à peu près, et acceptons l'imperfection, le médium, lui, avec ses logiciels experts, son traitement de la forme, nous pilote dans un monde à la fois malléable et rigoureux. Formalisation exemplaire pour l'esprit humain, il « formate » en quelque sorte notre pensée qui réclame alors, à l'instar du modèle, une plus grande exigence en matière de raisonnement et de mise en forme d'elle-même. La réflexivité cognitive et la formalisation informatique, comparables au travail théâtral vers la clarté, la rigueur, l'épure, pallient ce que les logiciens nomment le «manque à signifier» de nos activités quotidiennes floues et nouées.

En ce qui concerne l'apport de la technologie au théâtre proprement dit. il apparaît surtout dans l'interactivité, comme feedback instantané entre l'homme et la machine, 
pour s'ajouter à l'interaction des êtres entre lesquels existe toujours une relation plus ou moins réversible. Influencée par la mode de l'interactivité, la représentation du vivant incorpore de plus en plus l'innovation technologique et peut s'orienter vers une organisation totalement holographique permettant à des personnages animés d'interpréter un rôle dans un espace virtuel commun. À ce stade, un individu, à la fois spectateur et acteur, dialogue avec son propre clone. La spécialité de Paul SERMON allie ainsi la présence physique et la télé présence virtuelle. Son œuvre, Telematic Station, permet de dialoguer avec son double, dans une situation de communication hic et nunc. Si le théâtre, dans ce cas, procure au début un sentiment $d$ »inquiétante étrangeté ", il s'enrichit aussi de nouvelles expériences créatives étonnantes, à côté desquelles les expériences de théâtralité participante, de Brecht à Augusto Boal en passant par le Living théâtre, semblent appartenir au classicisme du genre.

\section{Vers une culture de l'interculturalité}

témoigne de phénomènes particuliers à notre époque qui transforment les points de vue sur les processus de médiation et d'échanges humains et techniques. Dans notre époque qui évolue plus vite que d'autres, on peut observer que l'heure n'est plus aux dualismes, aux dichotomies ou aux logiques de l'influence, une nouvelle épistémologie saurait faire émerger une logique de la combinaison, de la cohabitation des contraires, de la réconciliation des antagonismes, correspondant à de nouveaux modes de représentation, d'expression et de communication. La caractéristique de notre époque n'est-elle pas l'érosion des frontières entre l'homme et la femme, le bien et le mal, le corps et l'âme, l'homme et l'animal, le jeune et le vieux ? Des relations telles que : (sensible/intelligible), (sacré/profane), (information/communication), (humanisme/technologie), (émetteur/ récepteur), (réel/virtuel), (global/local), doivent maintenant s'envisager en termes d'alliance et de fusionnement. Chaque fois que l'homme actuel croise des formes, mêle des dispositifs, allie des éléments, il exprime du nouveau, invente des mondes, dans une logique de progrès. Cette question du métissage n'apparait-elle pas dans tous les domaines du pensable et du montrable, aussi bien dans les sciences, les médias, que dans l'art, le spectacle vivant, les cultures, et les imaginaires?

L'hybridité. jadis proscrite comme produit monstrueux d'un imaginaire délirant, mais que Novalis il y a deux siècles voulait, en un certain sens, réhabiliter, apparaît de plus en plus dans les idées comme dans les faits, comme un modèle à suivre. Accusé de suivre le sillage idéologique suspect de la globalisation, ce modèle - nouvelle utopie de la communication? - représente pourtant une troisième voie, entre l'uniformisation croissante qu'on constate dans certains domaines et l'exacerbation des particularismes identitaires. 
Hyperlittérature, technimage, info théâtralité, metaxu, bionique, cybionte, glocal. edutainment. infotainment, techno-nature, syncrétisme religieux, aïoli musical, mixages alimentaires, amalgames ritualistes, synthèses vestimentaires, sont des formes d'hybridité parmi d'autres. En tant que telles elles représentent de nouveaux objets d'étude, tout autant que l'attitude mentale qui les génère, que les dispositifs qui les animent, que les nouveaux sens qui s'en dégagent.

Elles pourraient par conséquent donner lieu à une grande problématique commune, originale, de recherche et d'enseignement qui pourrait enrichir le domaine d'étude des Sciences de l'Information et de la Communication, lieu ondoyant de rencontre des connaissances. Et si une «symbiologie » s'imposait sous peu comme une science nouvelle et fédératrice, portant son regard vers ce monde pluriel, chargé des valeurs postmodernes de pluralisme, d'hétérogénéité, de fragmentation, d'anachronisme. Cette science constituerait une approche pertinente du nouveau mythe englobant de nos sociétés, celui du grand melting pot culturel où toute prise de parole devient légitime et revendiquée, où le mélange des styles et des genres devient la règle, celui, en définitive, d'une culture de l'interculturalité.

\section{BIBLIOGRAPHIE}

Actes du Colloque, Les Technologies de l'Information et de la Communication : Pour quelle société?

Université de Technologie de Compiègne, 1997.

APPADURAI A., Cultural Dimensions of Globalization. Payot, 1996.

BALPE J-P., L'imagination informatique de la littérature, Colloque de Cerisy, L'Imaginaire du Texte. PUV, 1991

BARTHES R., Littérature et signification. Essais critiques. Seuil 1964.

CARO S. et BETRANCOURT M.. Ergonomie des documents techniques informatisés... approches cognitives et ergonomiques des hypermédias. Hermès. 2, 1998.

CLEMENT J., Fiction interactive et modernité, dans Informatique et Littérature, Revue Littérature, $\mathrm{n}^{\circ}$ 96, Larousse, 1994

Colloque de Cerisy, L'Imaginaire du Texte, PUV, 1991.

FICK JM, Les TIC : Un avenir pour les littéraires. Communication et Langages, $\mathrm{n}^{\circ} 124$, Armand Colin, 2000.

LAUFER R., SCAVETTA D., Texte, hypertexte, hypermédia. PUF. Que Sais-je?

LAUREL B., Computers and Theatre. Wesley Publishing Compagny, 1983.

LEVY P.. Essai sur la cyberculture : l'universel sans totalité. Rapport au conseil de l'Europe

http://hypermedia.univ-paris8.fr/pierre/cyberculture/cyberculture.html. 


\section{NOTES}

1. Camilleri C, Cultures et stratégies: ou les mille manières de s'adapter. Sciences Humaines. $\mathrm{n}^{\circ} 16$. 1992.

2. Prédiction de Daniel Bougnoux dans Sciences de l'information et de la communication. Textes Essentiels. Larousse. 1993

3. Carpentier J.B., Enjeux et problématiques d'une mutation annoncée. Actes du Colloque: Les Technologies de l'Information et de la Communication: Pour quelle société ? Université de Technologie de Compiègne. avril 1997.

4. Cyrulnik B.. L'ensorcellement du monde. Odile Jacob. 1997.

5. Cf. De Rosnay J.. L'homme symbiotique. Regards sur le troisième millénaire. Seuil. 1995.

6. Démocrite et lu philosophie épicurienne.

7. Cf. Lévy P.. L'Universel sans totalité, essence de la cyberculture, Actes du Colloque: Les technologies de l'Information et de la Communication : Pour quelle société ? op.cit..

8. Guillaume M., L'empire des réseaux. Descartes et Cie. 1999

9. Jaillissement de l'esprit, ordinateurs et apprentissage. Flammarion, coll. Champ. Paris, 1989.

10. Cf. Fick J.M., Les TIC : un avenir pour les littéraires. Communication et langages. $n^{\circ} 124$. juin 2000.

11. Simondon G., Du mode d'existence des objets techniques. Aubier. 1969.

12. Cf.Laufer R., Scavetta D.. Texte, hypertexte, hypermédia. PUF. Que Sais-je?

13. Poétique.

14. Baudin G. Le prétexte au désœuvrement, dans L'imagination informatique de la Littérature. Colloque de Cerisy. L'Imaginaire du Texte. 1991. p.99.

15. Interview à Libération du jeudi 30 novembre 1989.

16. L'article de Vannevar Bush, paru en 1945. présentant MF.MEX. premier concept d'hypertexte dont le but était l'accès facile à la masse des connaissances humaines, s'intitulait « As we May Think » (Selon notre façon de penser).

17. Barthes, cité par Anne-Marie Duquet dans «A propos de quelques implications artistiques de l'interactivité », dans Mutations de l'image, Paris. 1993.

18. Collection 2003. www.anacoluthe.fr

19. Clément J. et Laufer R., Revue littérature $n^{\circ} 96$, p. 5

20. Balpe J.P., L'imagination informatique de la littérature. Colloque de Cerisy. L'Imaginaire du Texte. PUV, 1991

21. Balpe J.P., La tentation de l'infini, Univ. Paris VIII.

22. Caractéristiques précisées par Jean Clément. Fiction interactive et modernité, dans Informatique et Littérature. Revue Littérature. $n^{\circ}$ 96. Larousse. 1994.

23. «Privé de son attrait principal qu'était le personnage, le roman moderne a cessé d'être « une aventure, une volonté et un destin » pour devenir " une technique et un mythe ». L'hypertexte... est plus qu'une nouvelle technique romanesque, il est une technologie nouvelle d'écriture et de lecture. Comme le roman moderne, il est aussi porteur d'un mythe, celui d'un univers déconstruit, disséminé, fragmentaire et discontinu [...] il offrirait dans ce cas une nouvelle chance à la littérature » : Clément J., Fiction interactive et modernité, op.cit..

24. Derrida J., De la grammatologie, Paris. Minuit. 1967.

25. Levy P., Essai sur la cyberculture: l'universel sans totalité: Rapport au conseil de l'Europe : http://hypermedia.univ-paris8.fr/pierre/cyberculture/cyberculture.html

26. Deleuze G., L'Image-Temps, Minuit. Paris.

27. Renaud-Alain A., L'image sans gravité, la forme image aux risques de l'information. Revue d'esthétique. 25. 1994. Sur les conséquences du passage de l'analogique au numérique, voir, entre 
autres. Couchot E., Images. De l'optique au numérique. Hermès. 1988. Réseaux. nº61. Vers une nouvelle pensée visuelle.

28. Quéau P., Metaxu, théorie de l'art intermédiaire. INA. La Découverte. 89.

29. The legible city.

30. Expression de Werner Herzog, entretiens avec Wim Wenders. Logique des images. L'Arche.

31. Cf. l'ouvrage de Brenda Laurel. (Computers and Theatre. Wesley Publishing Compagny. 1983) qui met en évidence pour la première fois le rapprochement.

32. B.O. $\mathrm{N}^{\circ} 14.8$ avril 1999.

33. Colin M., «Cinéma, télévision, cognition ». Nancy. Presses Universitaires. 1992

34. Cf "Vers une classification des procédés d'interactivité par niveaux carrélés aux données». A. Durand. J.M. Laulin. S. Leleu-Merviel, Hypertextes et hypermédias. I. Hermès. 1997.

35. Cf. l'enquête de Stéphane Caro et Mireille Bétrancourt. Ergonomie des documents techniques informatisés,... approches cognitives et ergonomiques des hypermédias. Hermès. 2. 1998.

36. Barthes R.. Littérature et signification. Essais critiques. Seuil 1964).

37. Barthes R.. op.cit.

38. Jouvet Louis. Témoignages sur le théâtre. Flammarion, 1951.

39. D. Bablet. Svoboda. Lausanne. La Cité. 1970.

40. Le Théâtre et son double.

41. titre de paragraphe de l'ouvrage d'Anne Ubersfeld. L'école du spectateur. Editions sociales. 1968

42. Cf Roger Caillois Les jeux et les hommes, Gallimard.

43. Cultural Dimensions of Globalization (Les conséquences culturelles de la globalisation. Payot. 1996).

\section{RÉSUMÉS}

L'échange interculturel n'apporte pas de désillusion dans des domaines où la pensée et l'imagination rencontrent avec bonheur des langages et des logiques informatiques et donnent alors naissance à des formes nouvelles, originales et rayonnantes. Prenant appui sur des exemples de mariage réussi de l'homme avec la machine, cet article fait entrevoir l'avènement dans nos sociétés d'une véritable culture de l'interculture. Elle est la conséquence d'une circulation mondiale et dynamique d'informations, d'images, de messages, dominée par des logiques de combinaison et de métissage entraînant de nouveaux modes de représentation, d'expression et de communication.

Intercultural exchanges are not a source of disappointment in fields within which creative thought happily mixes with computer languages and logic, in order to give birth to original and mind-challenging new forms of communication. Using examples illustrating harmonious associations between men and machines, this article anticipates the birth of a genuine cultural form, springing from new intercultural exchanges within our society. This new cultural form is the result of a dynamic, world-wide exchange of information, images and messages, prominently characterized by a combination of various types of logic, along with cultural cross-fertilization, thus promoting new forms of representation, expression and communication. 
INDEX

Mots-clés : artefact, culture humaniste, culture technologique, hyperlittérature. technimage, info théâtralité, métissage, nouvelles humanités, symbiologie

\section{AUTEUR}

\section{JEAN-MARC FICK}

Jean-Marc Fick est Maître de Conférences en Sciences de l'Information et de la Communication à l'Université de Bourgogne. Il dirige un département SIC au sein de l'UFR de Lettres et Philosophie et préside la Commission Culture de son université. Depuis son doctorat en 91, consacré à l'analyse du théâtre comme système de communication, il s'est engagé principalement dans l'étude des rapports de l'homme avec les technologies du numérique, impliquant les effets d'influence sur les comportements, les usages, les imaginaires, les symboliques. CV détaillé sur : http://www.u-bourgnone.fr/DESSEUROMEDIAS/JMFick.htm 